\title{
Enhanced Incretin Effects of Exendin-4 Expressing Chimeric Plasmid Based On Two-Step Transcription Amplification System with Dendritic Bioreducible Polymer for the Treatment of Type 2 Diabetes
}

\begin{abstract}
Keywords: Type 2 diabetes; TSTA system; Chimeric Exendin-4 polyplex; PAM-ABP dendritic polymer

Abstract

Glucagon-like peptide 1 (GLP-1) agonist, exenxdin-4, is currently being advanced as a promising diabetes remedy via a variety of incretin actions similar with GLP-1. In this study, we investigated an effective anti-diabetic therapy via exendin-4 expressing chimeric plasmid based on two-step transcription amplification (TSTA) system with dendrimer-type bioreducible polymer for more improved incretinbased gene therapy. Arginine-grafted poly (cystaminebisacryla midediaminohexane) (ABP)-conjugated poly (amido amine) (PAMAM) dendrimer (PAM-ABP) was used as gene camier. PAM-ABP/chimeric DNA polyplex was markedly elevated exendin-4 expression in ectopic cells as well as increased insulin production through an enhanced activation of protein kinase $K$ (PKA) induced by up-regulation of exendin-4-stimulated cyclic adenosine monophosphate (CAMP) in pancreatic $\beta$-cell. Consistent with these results, intravenous administration of PAM-ABP/chimeric DNA polyplex improved glucoregulotory effects, as well as increased insulin secretion by high expression of exendin-4 in blood in type 2 diabetic mice with no any toxic ity. Our exendin- 4 system can be attributed to provide a potentia diabetes therapeutic agent for improved incretin gene therapy.
\end{abstract}

\section{Introduction}

Most of all diabetes cases are applicable to type 2 diabetes mellitus $[1,2]$. Type 2 diabetes is a metabolic disorder which is characterized by high blood glucose (hyperglycemia) and by either the body does not produce enough insulin or the cells ignore the insulin in peripheral tissues (insulin resistance) [3-5].

At recent, exendin-4, glucagon-like peptide 1 (GLP-1) receptor agonistis highlighted as promising therapeutic agents and has been applied in clinical fields as exocrine hormone for the treatment of type 2 diabetes. It is well-known that exendin- 4 has a potent incretin effects such as enhancing glucose-dependent insulin secretion, suppressing glucose-dependent glucagon secretion, slowing gastric emptying, reducing food intake, stimulating $\beta$-cell health $[2,6-8]$. Besides, exendin- 4 plays a roles as an increase in glucose transport in muscle and glucose transporter expression and glycogen synthesis in liver $[8,9]$, as well as the improvement of heart function against

\section{Journal of}

\section{Gene Therapy}

\section{Pyung-Hwan Kim ${ }^{1}$, Minhyung Lee ${ }^{2}$, Kihoon Nam ${ }^{1}$ and Sung Wan Kim ${ }^{1,2 \dagger}$}

${ }^{1}$ Center for Controlled Chemical Delivery, Department of Pharmaceutics and Pharmaceutical Chemistry, University of Utah, Salt Lake City, UT 84112, USA

${ }^{2}$ Department of Bioengineering, College of Engineering, Hanyang University, Seoul, South Korea

\section{Address for Correspondence}

Dr. Sung Wan Kim, Center for Controlled Chemical Delivery, Department of Pharmaceutics and Pharmaceutical Chemistry, University of Utah, Salt Lake City, UT84112, USA, Tel: +1-801-581-5022; Fax: +1-801-581-7848; Email: SW.Kim@pharm.utah.edu

Copyright: (c) $2013 \mathrm{Kim}$ P, et al. This is an open access article distributed under the Creative Commons Attribution License, which permits unrestricted use, distribution, and reproduction in any medium, provided the original work is properly cited.

Submission: 22 October 2013 Accepted: 20 November 2013

Published: 07 December 2013

myocardial infarction [10] and the enhancement of central nervous system in brain [11].

While exendin- 4 is capable of acting as incretin hormone to diabetes, its clinical application is currently restricted to twice a daily or once a week administration [3]. To maintain the normal glucose levels in patient with type 2 diabetes, re-treatment is required. However, frequent administration causes unwanted adverse effects such as nausea, emesis, anti-exendin- 4 antibodies $[2,12]$.

Accordingly, new approaches are required for prevention and therapy of diabetes. Currently, the usage of viral vector such as adenovirus (Ad), adeno-associated virus (AAV), and lentivirus [1315] and peptide modification [16-18] to increase the activity and stability of exendin-4 in vivo have been investigated to maintain the ability of the long-acting exendin- 4 and extended therapeutic duration. As other possibilities, incretin-based gene therapy was designed to improve therapeutic efficacy via long-lasting action after single administration $[19,20]$. Exendin-4 gene delivery system should be comprised two approaches; 1) plasmid system capable of providing a long-term release and expression in order to avoid frequently readministration to maintain the normal glucose levels and 2) longcirculating self-polyplex forming high biocompatibility gene carrier with therapeutic DNA.

In our recent report for the treatment of type 2 diabetes, we used arginine-grafted cyctaminebisacrylamide-diaminohexane polymer (ABP) as efficient gene carrier and the two-step transcription amplification (TSTA) system composed of p $\beta$-Gal4-p65 and pUASSP-exendin-4 contained with signal peptide (SP) for gene expression efficiency and secretion as efficient gene expression system [21]. In that paper, diabetic mice treated with ABP/TSTA-SP-exendin-4 polyplex showed an increase of exendin- 4 expression in ectopic tissues, controlled glucoregulotory effects and enhanced insulin secretion with no serious toxicity, leading to greater anti-diabetic effects and efficacies for the treatment of type 2 diabetic animals.

Technically, to induce optimum expression of transgene in TSTA system itself, two plasmids should be delivered into a same cell. Among exendin-4 polyplexes, some polyplexes might have both $\mathrm{p} \beta$ - 
Citation: Kim PH, Lee M, Nam K, Kim SW. Enhanced Incretin Effects of Exendin-4 Expressing Chimeric Plasmid Based On Two-Step Transcription Amplification System with Dendritic Bioreducible Polymer for the Treatment of Type 2 Diabetes. J Gene Ther 2013;1(1): 9.

ISSN: $2381-3326$

Gal4-p65 and pUAS-SP-exendin-4used for TSTA effect. However, it is unlikely that all complexes have two plasmids. Therefore, it may be useful to construct TSTA system into a plasmid, which has both Gal4-p65 and UAS-SP-exendin-4 expression cassettes. Accordingly, chimeric TSTA plasmid based on TSTA system was newly generated to induce the expression of more efficient exendin-4. In addition, ABP polymer used as efficient gene carrier in the previous study was synthesized with the poly (amido amine) (PAMAM) to produce higher molecular weight $\mathrm{ABP}$ and to improve the use of $\mathrm{ABP}$ in vivo [22].

In this study, we evaluated in vitro and in vivo anti-diabetic effects of polyplex by newly designed chimeric exendin- 4 expression system and dendritic PAM-ABP bioreducible polymer.

\section{Materials and Methods}

\section{Cell lines and Plasmid Construction}

NIT-1, insulinoma pancreatic $\beta$-cell line established from NOD/ Lt mice; HeLa, cervical cancer cells were purchased from the American Type Culture Collection (ATCC, Manassas, VA). HeLacells were cultured in Dulbecco's Modified Eagle's Medium (DMEM; GibcoBRL, Grand Island, NY) or NIT-1 cells were maintained in DMEM/ F12 (1:1) (Gibco-BRL) with 10\% fetal bovine serum (FBS; Gibco$\mathrm{BRL}$ ) and penicillin/streptomycin (Gibco-BRL) at $37^{\circ} \mathrm{C}$ with $5 \% \mathrm{CO}_{2}$.

Chimeric plasmid containing secretable exendin- 4 expression cassette was constructed based on TSTA system used in a previously experiment $[21,23]$. To create exendin- 4 expressing chimeric TSTA pDNA, UAS-SP-exendin-4 cDNA from pUAS-SP-exendin- 4 at BglII and $B a m H I$ was inserted into the BamHI of pSP72 plasmid. Then, UAS-SP-exendin-4 cDNA from pSP72-UAS-SP-exendin-4 at ClaI was inserted into $\mathrm{p} \beta$-Gal4/p65 at ClaI. Finally, two plasmids used in TSTA system were constructed into one vector. The construction of the plasmids was confirmed by restriction enzyme studies and direct sequencing. The plasmids were amplified in Escherichia coli strain DH5a, and purified by Qiagen plasmids Maxi Kits (Qiagen, Valencia, CA). The purity of the plasmids was measured byabsorbance at 260 and $280 \mathrm{~nm}$ and the quantity was determined with absorbance at $260 \mathrm{~nm}$. The plasmids were stored at $-20^{\circ} \mathrm{C}$ until use. Scheme 1 shows the difference and function concept between TSTA pDNA and newly constructed chimeric pDNA system for exendin-4 expression (Scheme 1A and 1B).

\section{Cationic reagents and materials}

Hyperbranched poly(ethylenimine) (bPEI, 25kDa) and 3-(4,5-dimethylthiazol-2-yl)-2,5-diphenyltetrazolium bromide(MTT) were purchased from Sigma-Aldrich (St. Louis, $\mathrm{MO})$. The characterization and synthesis of the arginine-grafted bioreducible poly (disulfide amine) (ABP) cationic polymer and PAM-ABP dendritic polymer were performed and validated as previously described [22,24] (Scheme 1C), respectively.

\section{Preparation and Treatment of Plasmid Polyplexes with a Cationic Polymers}

Polyplexes were formed by vortexing total $1.5 \mu \mathrm{g}$ of exendin- 4 DNA solution with an equal volume of polymer solution in serum free DMEM. In the case of TSTA system, two plasmids were used at 1:1 $\mathrm{p} \beta$-Gal4/p65/pUAS-SP-exendin-4. The DNA solution was added to the polymer solution. The polyplexes were allowed to stand at room temperature for $25 \sim 30$ minutes before application to cells in culture medium without serum.

\section{Size and zeta potential measurements of PAM-ABP/ chimeric DNA polyplex}

Polyplexes with different concentrations (1:1 to 1:40) in HEPES buffer (HEPES $20 \mathrm{mM}$, 5\% glucose, $\mathrm{pH} 7.4$ ) were formed for 30 minutes incubation at room temperature. After polyplexes formation, polyplex solutions were added in filtered-water to a final volume of $500 \mu \mathrm{L}$ before measurement, and then the average size and zeta potential of DNA/polymer polyplexes were examined with a Nano ZS (ZEN3600, Malvern Instruments) with a He-Ne ion laser beam (633 $\mathrm{nm}$, fixed scattering angle $90^{\circ}$ ) at $25^{\circ} \mathrm{C}$.

\section{Expression levels of exendin-4 secreted from HeLa cells}

HeLacells were seeded at about $60-70 \%$ confluence in 12 -well plates $\left(1 \times 10^{5}\right.$ cells/well $)$ one day before transfection with polyplexes at $37^{\circ} \mathrm{C}$ in a humidified $5 \% \mathrm{CO}_{2}$ incubator. At 24 hours before transfection, the medium from each well was exchanged with fresh serum-free medium, and then cells were transfected with polyplexes. The cells were incubated with polyplexes for 6 hours and then the polyplexes solution was removed, and fresh $10 \%$ serum-containing medium was added. After $48 \mathrm{hrs}$, the level of exendin- 4 in the culture media was determined by exendin-4 Enzyme Immunoassay Kit (Phoenix Pharmaceuticals, Inc., Burlingame, CA) according to the manufacturer's protocol.

Insulin levels produced by PAM-ABP/chimeric DNA polyplexin NIT-1 insulinoma cells

To assess the induction levels of insulin by chimeric DNA polyplex with PAM/ABP polymer in NIT-1 cells, culture medium

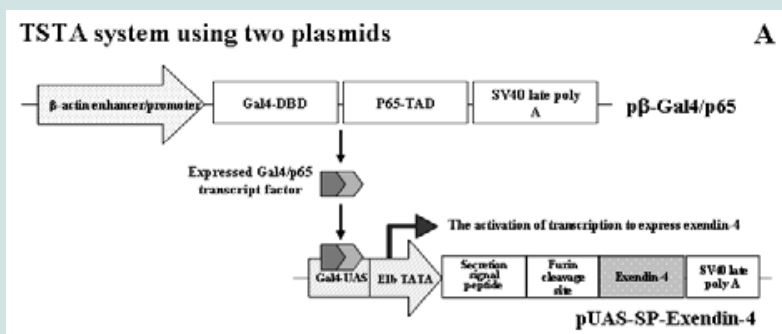

Chimeric TSTA system using one plasmid

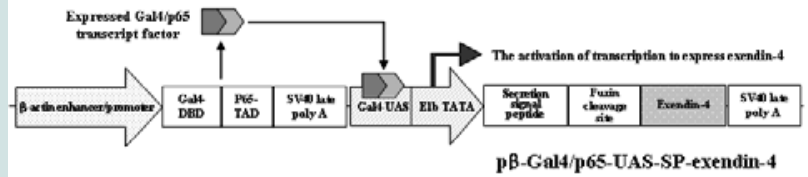

PAM-ABP (ABP-conjugated PAMAM)

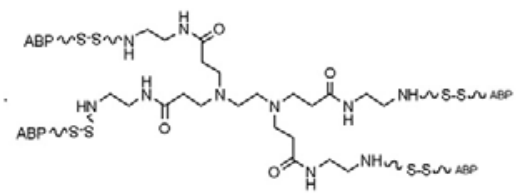

Scheme 1: Structure of TSTA pDNA consisted of two plasmids (A) and newly constructed chimeric TSTA pDNA (B) as efficient gene expression system and ABP-conjugated PAMAM (PAM-ABP) polymer (C) as efficient gene carrier. 
Citation: Kim PH, Lee M, Nam K, Kim SW. Enhanced Incretin Effects of Exendin-4 Expressing Chimeric Plasmid Based On Two-Step Transcription Amplification System with Dendritic Bioreducible Polymer for the Treatment of Type 2 Diabetes. J Gene Ther 2013;1(1): 9.

containing exendin- 4 secreted from HeLa cells was exchanged with medium of NIT-1 cells prepared in 12-well plates. At 5 hours after change, insulin level secreted in supernatant was determined by Rat/ mouse insulin ELISA (Millipore, Billerica, MA) according to the manufacturer's protocol.

The evaluation of intracellular signaling pathway in exendin-4-stimulated pancreatic $\beta$-cells

To examine the levels of exendin-4-stimulated 3'-5'-cyclic adenosine monophosphate (cAMP) and cAMP-induced protein kinase A (PKA) activated by exendin- 4 expressed by each polyplexes, culture medium containing exendin- 4 secreted from HeLa cells was exchanged with medium of NIT-1 cells prepared in 12-well plates. At 5 hours for cAMP assay and at $7 \mathrm{hrs}$ for PKA assay after change, cAMP and PKA levels in cell lysates was measured by cAMP Parameter Assay Kit (R\&D systems, Minneapolis, MN) and PKA kinase activity kit (Enzo life sciences, Farmingdale, NY) according to the manufacturer's protocol, respectively.

\section{Animal care}

All animal studies were performed according to established NIH and University of Utah Animal Care Committee guidelines. To examine actual incretin effects of chimeric DNA polyplex with PAM-ABP polymer in diabetic mice, diet-induced obese (DIO) male C57BL/6J mice (Jackson Laboratory, Bar Harbor, ME), 8 weeks of age, were used in same condition and management in our previous experiment [21]. Mice were fed with a high-fatand high-calorie diet (60 kcal\% fat, D12492; Research Diet, NewBrunswick, NJ) for 1 month.

\section{In vivo Anti-Diabetic study}

The animals ( $n=5$ per group) were randomized as pre-glucose levels before injection and then treated by intravenous single administration with PBS, chimeric DNA only, $25 \mathrm{~K} \mathrm{PEI/chimeric}$ DNA, ABP/chimeric DNA, PAM-ABP/TSTA DNA, and PAM$\mathrm{ABP} /$ chimeric DNA polyplexes of $60 \mu \mathrm{g}$ exendin-4 DNA with $w: w$ ratio of 1:5 DNA/polymer. Blood samples were obtained from the retro-orbital vein after anesthesia at days $0,3,6,9,12$ and 15 after injection to measure blood glucose, exendin- 4 expression level, and insulin concentrations. Blood glucose levels were measured by a onetouch blood glucose meter (ACCU-CHEK sensor, Roche Diagnostics Corp., Indianapolis, IN).

Assessment of free fatty acid (FFA) levels in PAM-ABP/ chimeric DNA polyplex-treated mice

To evaluate whether exendin- 4 expressed by PAM-ABP/chimeric DNA polyplex can decrease FFA levels in DIO mice, mice were systemically injected one time with various polyplexes. At 3 days after-administration, mice were fasted for $6 \mathrm{hrs}$ and then serum FFA levels were assessed by serum/plasma fatty acid and glycerol detection kits (Zenbio, Inc., Research Triangle Park, NC).

\section{Hepatotoxicity in mice treated with $\mathrm{PAM}-\mathrm{ABP} /$ chimeric DNA polyplex}

Diabetic mice were injected intravenously with several compounding. Serum levels of aspartate aminotransferase (AST) and alanine transaminase (ALT) representing liver toxicity were then measured at 3 days post-injection by ALT or AST enzyme assay kit (Bioo Scientific co., Austin, TX) according to the manufacture's protocol, respectively.

\section{Statistical Analysis}

The data were expressed as mean \pm standard error of the mean (SEM). Statistical comparisons were made using Stat View software (Abacus Concepts, Inc., Berkeley, CA) and the Mann-Whitney test (non parametric method). Differences were considered statistically significant when $P$ values were less than 0.05 .

\section{Results}

\section{Polyplexes characterization according to PAM-ABP weight ratios}

After the formation of polyplex with exendin-4 expressing chimeric TSTA DNA and PAM-ABP polymer, the average sizes and the surface-charge values of chimeric DNA polyplexes according to PAM-ABP ratios were measured by DLS assay. Chimeric DNA in total $1.5 \mu \mathrm{g}$ DNA was formed the polyplexes from 1:1 to $1: 40 \mathrm{w} / \mathrm{w}$ ratios DNA to PAM-ABP polymer. As the results shown in Figure 1, all polyplexes except polyplex at 1:1 ratio were stably formed at below $70 \mathrm{~nm}$ in size with weight ratios above 1:2. These results mean that PAM-ABP/chimeric DNA polyplex with diameter 50-100 nm can induce rapid internalization to target cells [25]. Also, zeta-potential values of the polyplexes were measured to up to about $+30 \mathrm{mV}$ from 1:5 ratio by strong positive charge of PAM-ABP surface [22], suggesting that the change of surface charge was formed condensed polyplex depending on the increase of PAM-ABP ratios. These results indicate that negatively charged chimeric TSTA pDNA can form the polyplex with the positively-charged PAM-ABP polymer.

Increased effects of chimeric DNA system with PAM-ABP polymer on exendin-4 expression level and stimulatedinsulin secretion

Next, to test whether the polyplex with newly constructed exendin- 4 expressing chimeric DNA and PAM-ABP polymer can actually induce and secret higher exendin-4 expressionin ectopic cells thanthat of TSTA system, HeLa cells were treated with various polyplexes combined with $25 \mathrm{~K}$ PEI (1:1 ratio), ABP (1:10 ratio), and

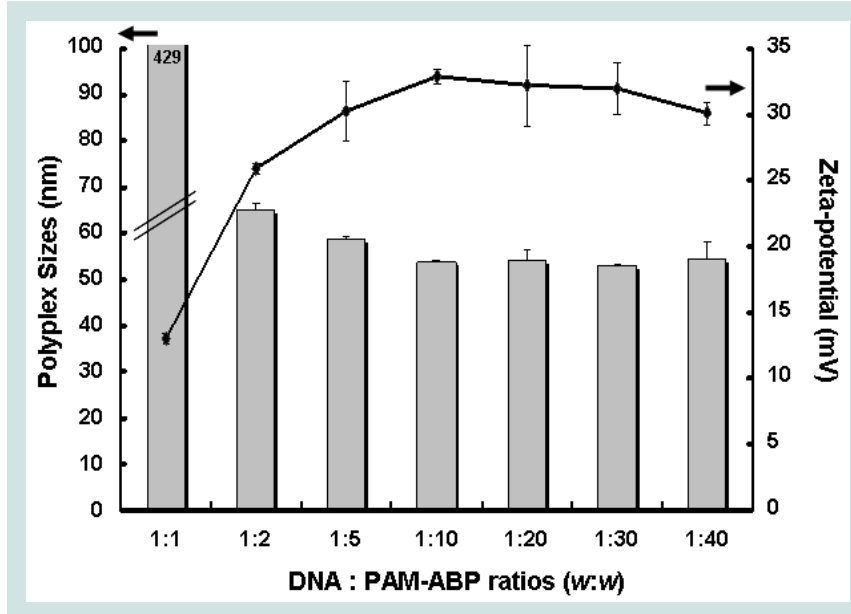

Figure 1: Characterization of chimeric TSTA pDNA polyplexes with PAMABP dendritic polymer. Size distribution and zeta-potential values were determined as the change in polyplex according to the increase of PAM-ABP polymer ratios from $1: 1$ to $1: 40$. Chimeric DNA was total amount of $1.5 \mu \mathrm{g}$ for all polyplexes. Data represent the means and standard errors of triplicate experiments. 
Citation: Kim PH, Lee M, Nam K, Kim SW. Enhanced Incretin Effects of Exendin-4 Expressing Chimeric Plasmid Based On Two-Step Transcription Amplification System with Dendritic Bioreducible Polymer for the Treatment of Type 2 Diabetes. J Gene Ther 2013;1(1): 9.

PAM-ABP (1:10 ratio) polymer and exendin-4 expressing TSTA (1:1 ratio $\mathrm{p} \beta$-Gal4/p65/ $\mathrm{pUAS-SP-exendin-4} \mathrm{plasmids)} \mathrm{or} \mathrm{chimeric} \mathrm{DNA}$ system. PAM-ABP polymer was determined a 1:10 $w / w$ ratio showing the best optimal high gene transfer efficiency and no cytotoxicity as observed in Supplementary Figure 1 and 2.

As shown in Figure 2A, exendin-4 was detected in cells treated with all groups. Chimeric DNA system treated cells showed higher exendin-4 expression than TSTA system in the comparison of each polymer. Also, the highest exendin- 4 expression was observed in
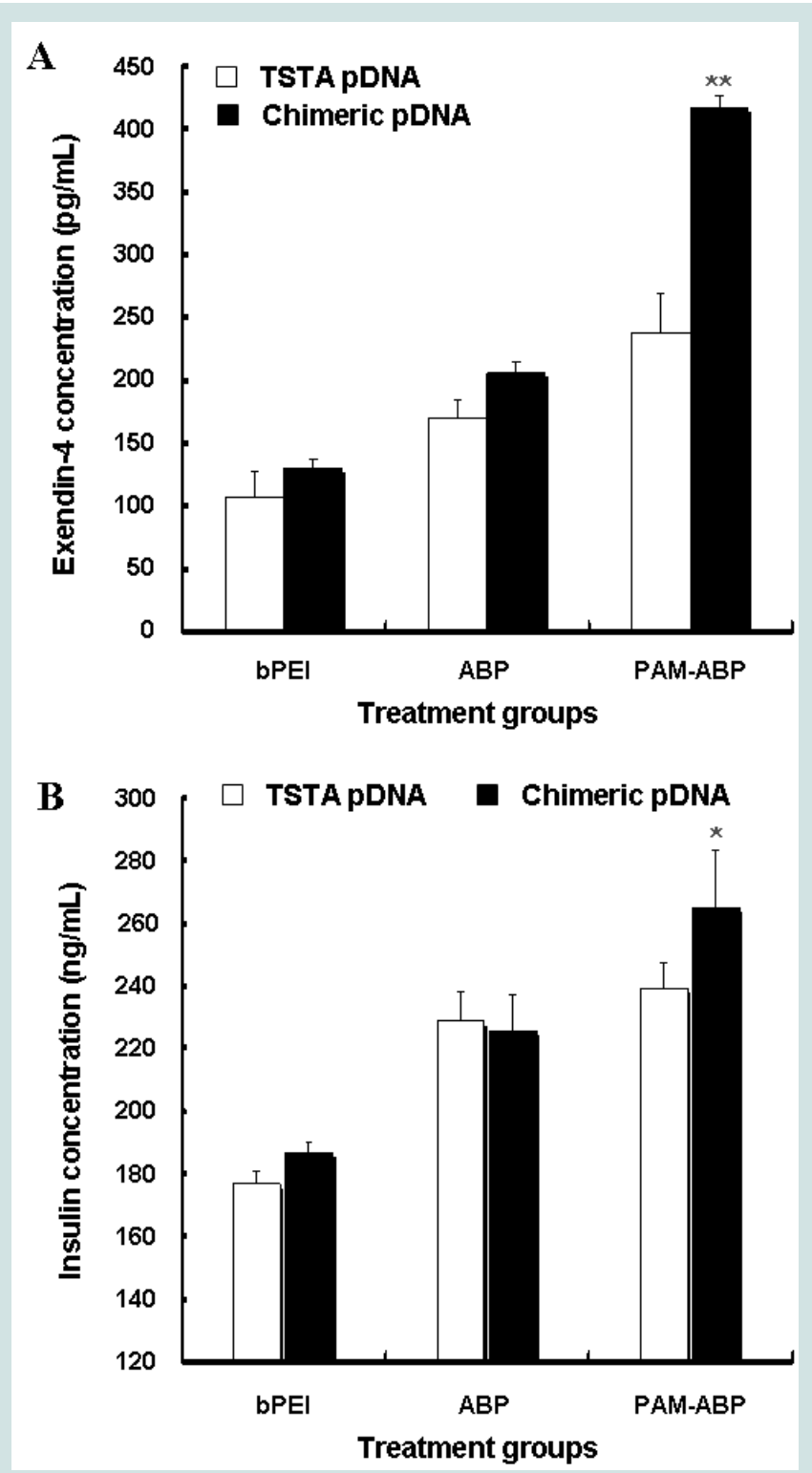

Figure 2: Increased exendin-4 expression in HeLa cells (A) and insulin induction in insulinoma NIT-1 cells (B) by PAM-ABP/chimeric DNA polyplex. (A) HeLa cells were treated with each polyplexes formed with total $1.5 \mu \mathrm{g}$ DNA at $1: 10 \mathrm{w} / \mathrm{w}$ ratio for $6 \mathrm{hrs}$. At this time, TSTA pDNA used 1:1 p $\beta-G a l 4$ p65/pUAS-SP-exendin-4 ratio decided previously.

${ }^{* *} \mathrm{P}<0.02$ versus PAM-ABP/TSTA DNA polyplex treatment. (B) Culture medium including secreted exendin- 4 from HeLa cells was treated to NIT-1 cells for 5 hrs and then insulin levels secreted in media were assessed. * $P<$ 0.05 versus $A B P / c h i m e r i c$ DNA polyplex-treated group. All data represent the means and standard errors of triplicate experiments.
HeLa cells treated with PAM-ABP/chimeric DNA polyplex. The levels of exendin- 4 expression in the cells treated with $\mathrm{PAM}-\mathrm{ABP} /$ chimeric DNA were 3.2- (or) 2-fold higher than those of cells treated with $\mathrm{PEI} /$ chimeric DNA or ABP/chimeric DNA, respectively. Moreover, PAM-ABP/chimeric DNA polyplex showed 1.8-fold high exendin-4 expression compared with that of PAM-ABP/TSTA DNA polyplex. Taken together, these results suggest that chimeric DNA system with PAM-ABP polymer can efficiently induce an increased expression of exendin-4 compared with TSTA DNA system and mean that one vector chimeric DNA system contained both plasmids in TSTA system works well.

We then assessed whether exendin-4 expressed by chimeric DNA system and PAM-ABP polymer can induce the insulin secretion in NIT-1 pancreatic $\beta$-cells. The results of insulin induction in Figure 2B showed similar pattern with an increased expression of exendin- 4 in Figure 2A. PAM-ABP-treated NIT-1 cells were stimulated for much higher insulin secretion and induced the highest insulin production compared with the other groups. The insulin levels by PAM-ABP/ TSTA DNA polyplex was assessed lower than that of PAM-ABP/ chimeric DNA polyplex. These results demonstrate that chimeric DNA and PAM-ABP polymer system can improve the insulin secretion-inducing ability of exendin- 4 in vitro.

Up-regulation of cAMP pathway by chimeric TSTA DNA with PAM-ABP polymer in pancreatic $\beta$-cell for insulin secretions

Since the activation of signaling pathway in $\beta$-cells stimulated via exendin- 4 is one of major potential mechanisms for insulin secretion $[6,26]$. Thus, to assess whether exendin- 4 expressed by PAM-ABP/ chimeric DNA polyplex can stimulate $\beta$-cells for insulin secretion, NIT-1 insulinoma cells were treated with various exendin-4 formulations for 6 hours. As shown in Figure 3A, the exendin-4induced highest cAMP elevation was observed in NIT-1 insulinoma cells treated with PAM-ABP polymer $(14.1 \pm 0.5 \mathrm{pmol} / \mathrm{mL})$ compared with those of PEI $(7.92 \pm 1 \mathrm{pmol} / \mathrm{mL})$ or ABP $(12.9 \pm 0.1 \mathrm{pmol} / \mathrm{mL})$ polymer with chimeric DNA system. Indeed, cAMP level by chimeric DNA system was stimulated 1.4-fold higher in comparison with that of TSTA DNA system (Figure 3B). These results demonstrate that exendin- 4 expressed by chimeric TSTA DNA with PAM-ABP polymer induces up-regulation of cAMP in $\beta$-cells.

\section{PKA induction level by PAM-ABP/chimeric DNA polyplex}

The increased insulin production in $\beta$-cells may also be caused by increased activation of cAMP-stimulated downstream signaling for insulin secretion. To assess level of protein kinase A (PKA) activation known as exchange proteins among cAMP-mediated downstream signaling pathway by exendin- 4 on insulin secretion, NIT- 1 cells were treated with PAM-ABP/chimeric DNA or /TSTA DNA polyplexes. At 48 hours post-treatment, PKA level of cell lysates were measured by ELISA.

Consistent with up-regulation of cAMP by exendin- 4 expressed by chimeric DNA with PAM-ABP (Figure 3A,3B), a remarkable high elevation of PKA via cAMP-mediated signaling mechanism was observed around 1.3-fold higher in $\beta$-cells treated with PAM$\mathrm{ABP} /$ chimeric DNA polyplex (Figure 3C). This result means that exendin- 4 expressed by chimeric DNA with PAM-ABP is functional and engaged downstream signal transduction pathway, implying that the increase of insulin production may be caused by the increment of PKA activation via exendin-4-induced cAMP. 
Citation: Kim PH, Lee M, Nam K, Kim SW. Enhanced Incretin Effects of Exendin-4 Expressing Chimeric Plasmid Based On Two-Step Transcription Amplification System with Dendritic Bioreducible Polymer for the Treatment of Type 2 Diabetes. J Gene Ther 2013;1(1): 9.
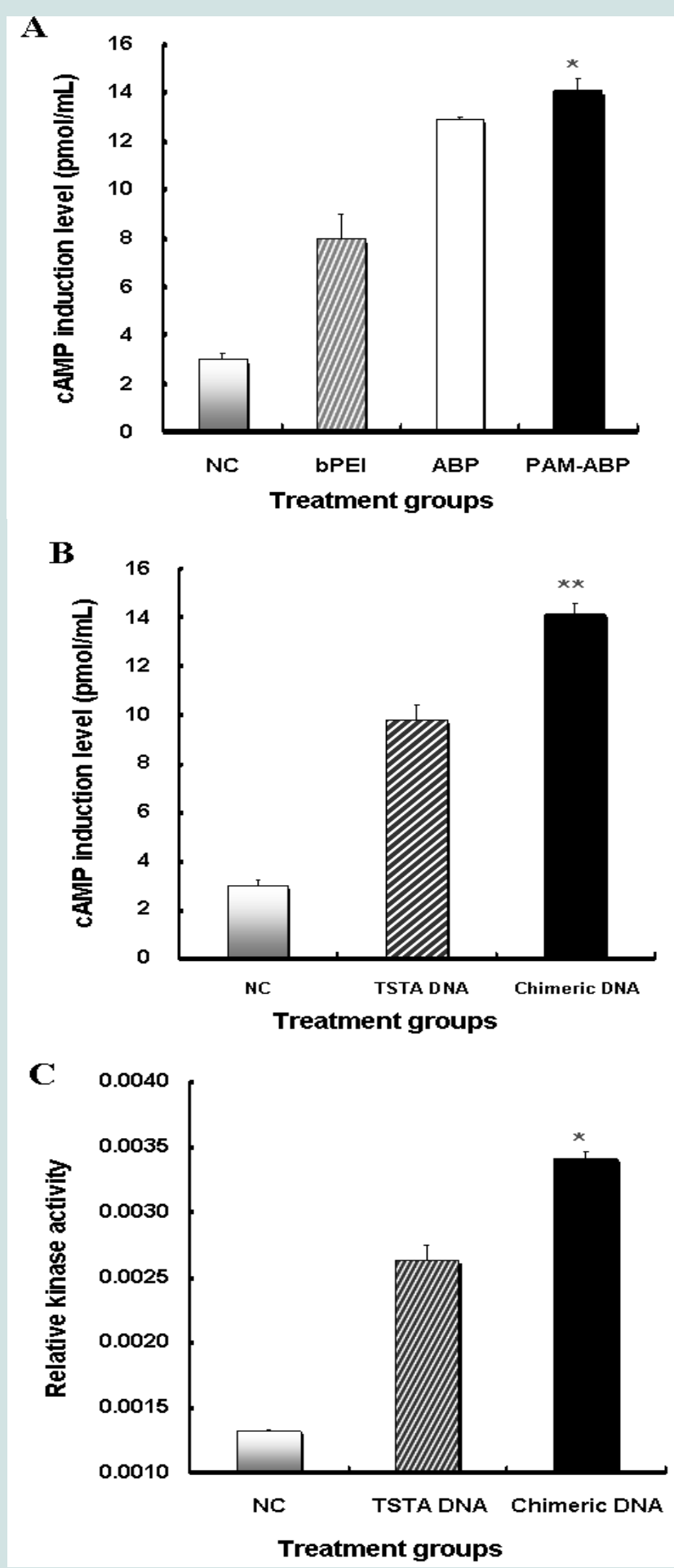

Figure 3: The evaluation of functional ability in exendin-4-stimulated $\beta$-cells for insulin secretion. (A) cAMP elevation in $\beta$-cells activated exendin-4 according to each polymer. ${ }^{*} \mathrm{P}<0.05$ for comparison of $\mathrm{PAM}-\mathrm{ABP} /$ chimeric DNA polyplex against $25 \mathrm{~K} \mathrm{PEl} /$ chimeric DNA polyplex. (B) The induction levels of cAMP in $\beta$-cells activated exendin- 4 according to TSTA pDNA and chimeric TSTA pDNA system. ${ }^{*} \mathrm{P}<0.02$ for comparison of TSTA DNA versus chimeric DNA polyplex when used PAM-ABP polymer. (C) The levels of PKA activation in response to up-regulation of exendin-4-induced cAMP. ${ }^{*} \mathrm{P}<$ 0.05 for comparison of PAM-ABP/TSTA polyplex versus PAM-ABP/chimeric DNA polyplex. Each data point represents the means and standard errors of triplicate experiments.
Enhanced effects of PAM-ABP/chimeric DNA polyplex on blood glucose availability in diabetic mice

Exendin-4 as exocrine hormone is well known to lower body glucose concentration as one of the main physiological actions against diabetes. Thus, we examined whether exendin- 4 delivered by PAM$\mathrm{ABP} /$ chimeric DNA polyplex can induce glucose homeostasis. DIO diabetic mice were treated one time with various polyplexes including $\mathrm{PAM}-\mathrm{ABP} /$ chimeric DNA polyplex via systemic administration.

As shown in Figure 4A, DIO mice treated with PAM-ABP/ chimeric DNA polyplex showed the lowest glucose level compared with the others groups for the entire experiment time, although the blood glucose of mice was fluctuated. These values gradually returned to normal level from third day to fifteenth day after polyplexes injection

Significant less blood glucose was induced in response to intravenous administration of PAM-ABP/chimeric DNA (113 \pm $22.7 \mathrm{mg} / \mathrm{dL}$ ) compared with those of PEI/chimeric DNA (154.3 \pm $10.2 \mathrm{mg} / \mathrm{dL}), \mathrm{ABP} /$ chimeric DNA $(148.3 \pm 29.2 \mathrm{mg} / \mathrm{dL})$, or PAM$\mathrm{ABP} / \mathrm{TSTA}(141 \pm 22.6 \mathrm{mg} / \mathrm{dL})$ at third day. In contrast, the control groups (NC and chimeric DNA only) remained under hyperglycemia condition with no significant change in their blood glucose levels $(233.8 \pm 13.2 \mathrm{mg} / \mathrm{dL}$ and $216.6 \pm 13.5 \mathrm{mg} / \mathrm{dL})$, respectively. Above all, $\mathrm{PAM}-\mathrm{ABP} /$ chimeric DNA polyplex-treated mice maintained longlasting glucoregulatory effects within normal therapeutic range. The results shown in Figure 4A demonstrate that exendin-4 expressed by chimeric DNA system and dendritic PAM-ABP polymer has the ability of greater glucose homeostasis to maintain normoglycemia in diabetic mice.

Improvements of insulinotropic effects by $\mathrm{PAM}-\mathrm{ABP} /$ chimeric DNA polyplex in diabetic mice

The enhancement of exendin-4-mediated glucoregulotory effects may be because of increasing insulinotropic effects. Therefore, we examined whether exendin- 4 expressed by chimeric TSTA system and PAM-ABP polymer actually has the insulinotropic effect in diabetic mice.

As shown in Figure 4B, insulin level in mice treated with PAM$\mathrm{ABP} /$ chimeric or /TSTA DNA polyplexes began to dramatically increase after injection, and then insulin concentration steadily decreased to the experimental period. The measured insulin levels were raised to $433.8 \pm 58.4 \mathrm{pmol} / \mathrm{L}$ for $\mathrm{PAM}-\mathrm{ABP} /$ chimeric DNA polyplex and $337.1 \pm 35.9 \mathrm{pmol} / \mathrm{L}$ for PAM-ABP/TSTA DNA polyplex compared with those of chimeric DNA only $(96.68$ $\pm 96.3 \mathrm{pmol} / \mathrm{L}$ ) at $3^{\text {rd }}$ day after single intravenous injection and then returned to basal levels. However, mice treated with PAM-ABP/ chimeric DNA or /TSTA polyplex still maintained high insulin levels in blood compared with those of DNA only or NC treated groups for the entire experiment time. Among them, better insulinotropic effect in response to exendin-4 was found around 1.3-fold higher in mice groups treated with $\mathrm{PAM}-\mathrm{ABP} /$ chimeric DNA system in comparison with PAM-ABP/TSTA DNA system. This result demonstrates that our system with chimeric DNA and dendritic PAM-ABP polymer have a potentiating effect of exendin- 4 on glucose-stimulated insulin secretion.

Increased exendin-4 expression in PAM-ABP/chimeric DNA system-treated diabetic mice

The improved incretin effects based on the enhanced glycemic 
Citation: Kim PH, Lee M, Nam K, Kim SW. Enhanced Incretin Effects of Exendin-4 Expressing Chimeric Plasmid Based On Two-Step Transcription Amplification System with Dendritic Bioreducible Polymer for the Treatment of Type 2 Diabetes. J Gene Ther 2013;1(1): 9.

control and increased insulinotropic effects shown in Figure 4A and $4 \mathrm{~B}$ may also be caused by increased exendin- 4 expression by PAM-ABP dendritic polymer and chimeric DNA system as incretin hormones.

To verify these facts, we evaluated the exendin- 4 expression levels in mice treated with $\mathrm{PAM}-\mathrm{ABP} /$ chimeric DNA polyplex. As shown in Figure 4C, the highest exendin-4 expression was observed in mice treated with $\mathrm{PAM}-\mathrm{ABP} /$ chimeric DNA polyplex from the $3^{\text {rd }}$ day after injection during experimental period and then gradually decreased. In the other hand, exendin-4 levels of PAM-ABP/TSTA DNA polyplex resulted in low exendin-4 expression compared with chimeric DNA system with PAM-ABP polymer. Among all treatment groups, exendin-4 levels of chimeric DNA polyplex with PAM-ABP were 1.5-, 1.3- or 1.2-fold higher than those in mice treated with $\mathrm{PEI} /$ chimeric DNA, ABP/chimeric DNA or PAM-ABP/TSTA DNA, respectively, at $3^{\text {rd }}$ day. After all, Figure $4 \mathrm{C}$ means that improvements of glucose disposal and insulin secretion are dependent on exendin-4 expression.

\section{Serum Free Fatty Acid (FFA) level in PAM-ABP/chimeric DNA-treated mice}

Free fatty acid (FFA) has been implicated to play an important role in insulin resistance in obese patients with type 2 diabetes. Moreover, elevated FFA levels is known to inhibit insulin-stimulated glucose clearance [27]. To address these facts, we sought to determine whether FFA levels in blood is associated with insulinotropic effects improved after administration of $\mathrm{PAM}-\mathrm{ABP} /$ chimeric DNA polyplex.

As shown in Figure 5A, all exendin-4 expressing DNA polylplexes with polymer induced efficient reduced FFA levels in diabetic mice. Moreover, the lowest FFA levels was observed in mice treated with PAM-ABP/chimeric DNA polyplex $(437.3 \pm 8.7 \mu \mathrm{mol} / \mathrm{L})$ compared to mice treated with PEI/chimeric DNA $(536.7 \pm 20.8 \mu \mathrm{mol} / \mathrm{L})$, $\mathrm{ABP} /$ chimeric DNA $(519.3 \pm 22.5 \mu \mathrm{mol} / \mathrm{L})$, whereas $\mathrm{NC}$ or chimeric DNA only groups showed high FFA levels $(613.3 \pm 14.7 \mu \mathrm{mol} / \mathrm{L}$ or $624 \pm 57.5 \mu \mathrm{mol} / \mathrm{L})$, respectively. In addition, exendin- 4 expressed by chimeric DNA induced 1.1-fold decreased level of FFA than that of TSTA DNA group $(460.7 \pm 14.6 \mu \mathrm{mol} / \mathrm{L})$ when used PAMABP polymer. Reduced FFA level elicited by PAM-ABP/chimeric DNA polyplex. These results implicate that exendin- 4 delivered by chimeric DNA and PAM-ABP polymer system has the property of reducing level of FFA, leading to both improved insulinotropic and glucoregulatory effects and the protection against lipotoxicity.

\section{Liver toxicity evaluation in PAM-ABP/chimeric DNA- treated mice}

The particle size less than $100 \mathrm{~nm}$ is able to cause liver toxicity by through from blood to endothelial fenestrae in liver sinusoids [28]. Therefore, we analyzedany toxicity associated with the plasmid infusion and sizes of exendin-4 expressing DNA polyplexes.

In the result of Figure 5B, the highest AST and ALT levels were found in mice treated with $\mathrm{PEI} /$ chimeric DNA polyplex after injection. In contrast, serious increase in ALT and AST levels were not observed in mice treated with chimeric DNA only, $\mathrm{ABP} /$ chimeric DNA, PAMABP/TSTA DNA, and PAM-ABP/chimeric DNA polyplexes. This result means that PAM-ABP polymer and plasmids coding exendin-4 did not induce polyplex-associated hepatic injury.

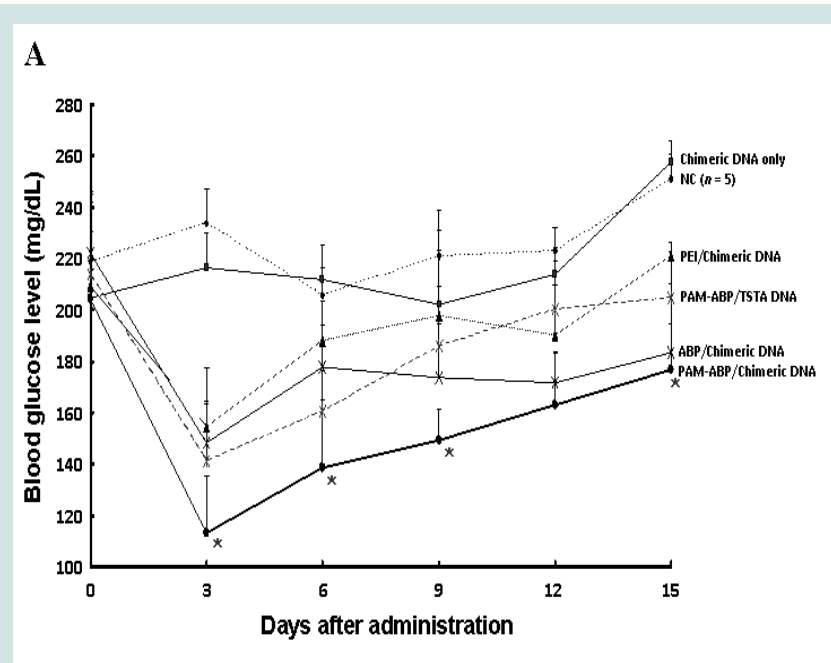

B

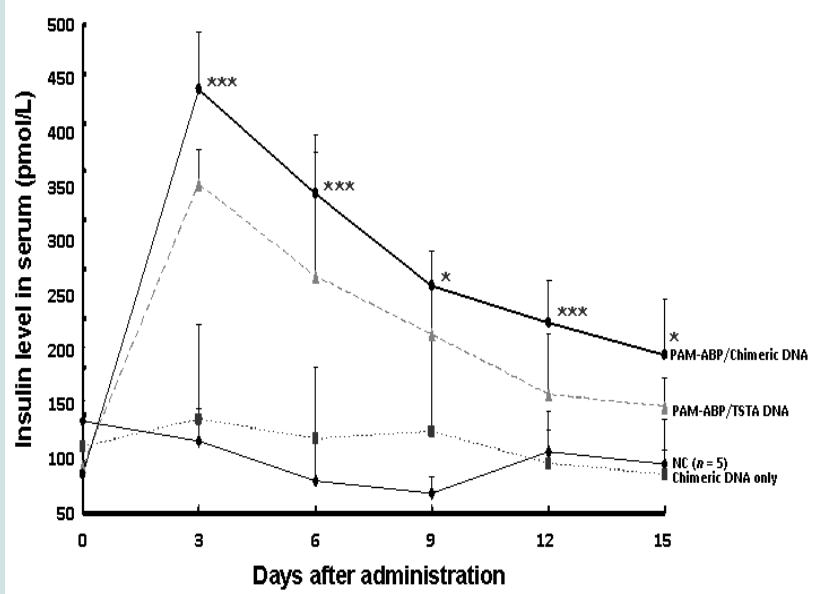

C

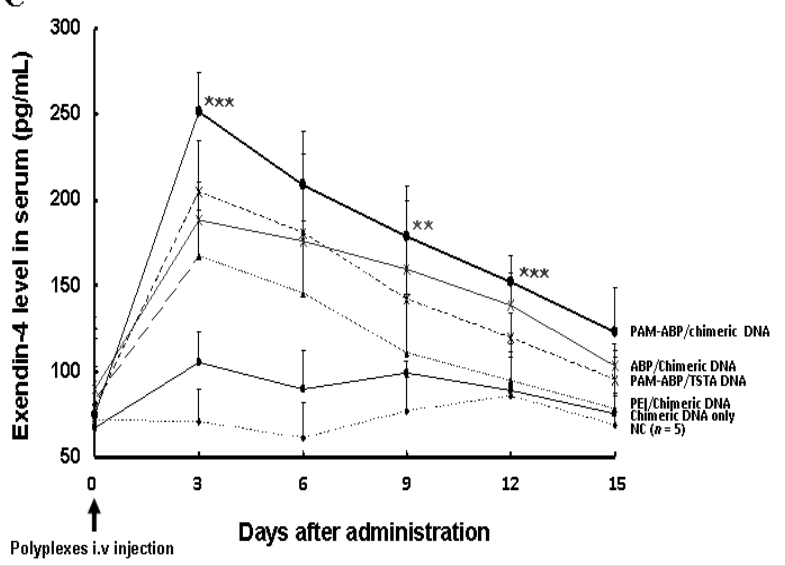

Figure 4: The anti-diabetic effects PAM-ABP/chimeric DNA polyplex after systemic administration. Glycemic control (A), insulinotropic effects (B), and expression levels of exocrine hormone $(C)$ in DIO diabetic mice treated with PAM-ABP/chimeric DNA polyplex. Mice received NC $(\bullet, n=5)$, chimeric DNA only $(\boldsymbol{\square}, n=5)$, PEI/chimeric DNA polyplex $(\boldsymbol{\Delta}, n=5)$, ABP/chimeric DNA polyplex $(X, n=5)$, PAM-ABP/TSTA DNA polyplex $(*, n=5)$, or PAMABP/chimeric DNA polyplex $(\bullet, n=5)$ on days $0,3,6,9,12$, and 15 . Data represent means $\pm S E .{ }^{*} P<0.05$ versus $P E I /$ chimeric DNA polyplex-treated group for $(A)$; ${ }^{*} P<0.05$ or ${ }^{* * *} P<0.01$ versus chimeric DNA-treated group for (B); ${ }^{* *} \mathrm{P}<0.02$ or ${ }^{* * *} \mathrm{P}<0.01$ versus $25 \mathrm{~K} \mathrm{PEl} /$ chimeric DNA-treated group for (C) 
Citation: Kim PH, Lee M, Nam K, Kim SW. Enhanced Incretin Effects of Exendin-4 Expressing Chimeric Plasmid Based On Two-Step Transcription Amplification System with Dendritic Bioreducible Polymer for the Treatment of Type 2 Diabetes. J Gene Ther 2013;1(1): 9.

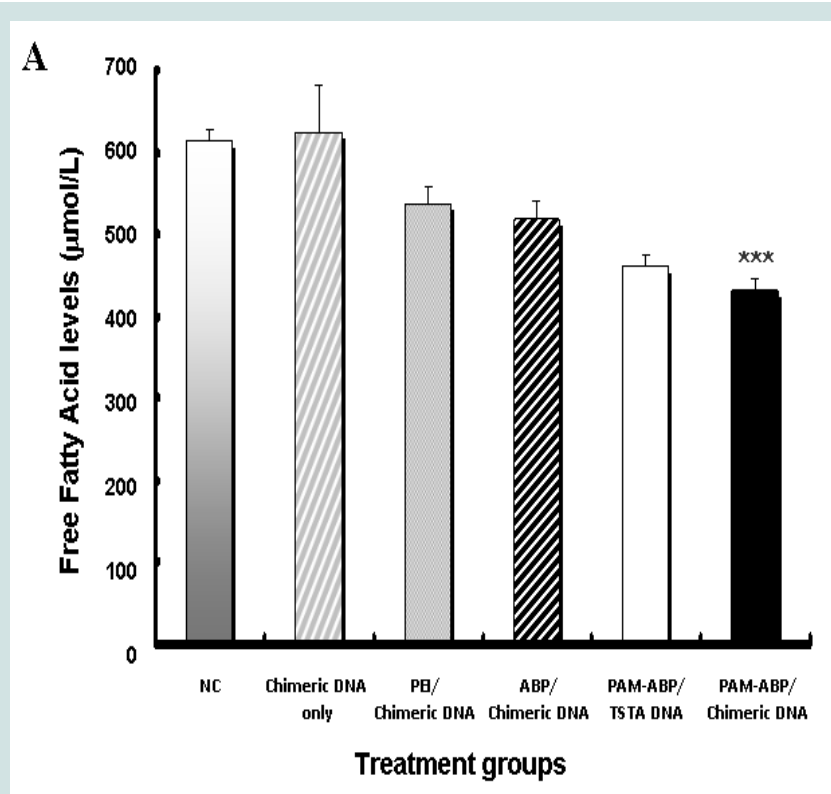

B

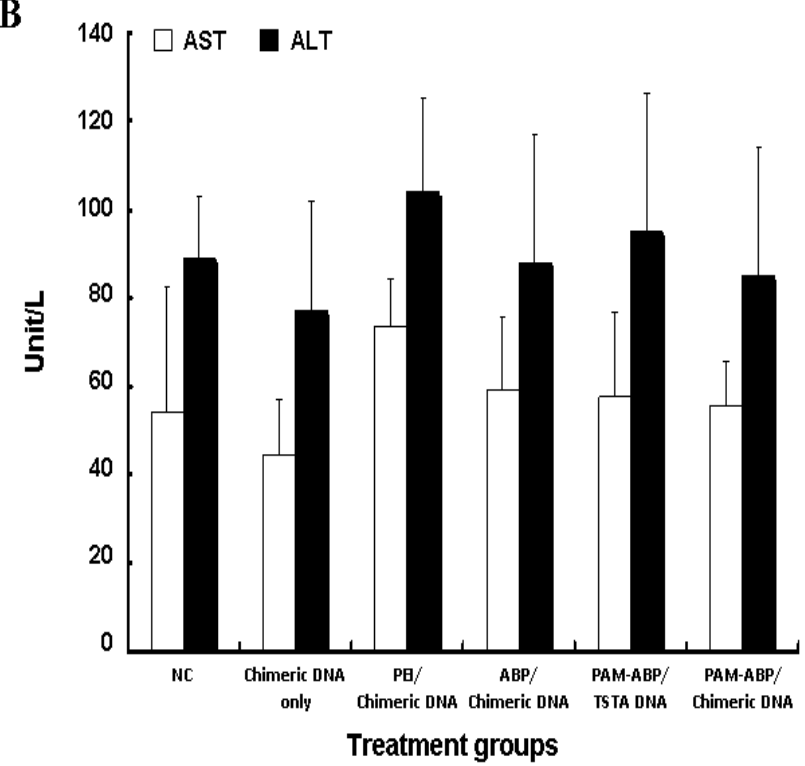

Figure 5: The assessment of FFA levels and hepatotoxicity related to PAM$\mathrm{ABP} /$ chimeric DNA polyplex upon intravenous administration in diabetic mice. (A) The reduced FFA levels in PAM-ABP/chimeric DNA polyplex-treated with mice. At 3 days post-systemic administration, mice were fasted for 6 hrs before sample harvest and then serum FFA levels were measured. ${ }^{* *} \mathrm{P}<$ 0.01 versus NC-treated group. (B) In vivo liver toxicity evaluation in mice treated with PAM-ABP/chimeric DNA polyplex. At 3 days after injection of various compounding, mice serum levels of $A L T$ and $A L T$ were analyzed. Data represent means $\pm S E$ and $n=5$ for each experimental condition.

\section{Discussion}

Exendin-4, glucagon-like peptide 1 (GLP-1) receptor agonist is well-known to play as important roles in enhancing glucosedependent insulin secretion, suppressing glucose-dependent glucagon secretion, reducing food intake, and slowing gastric emptying as well as enhancing $\beta$-cells health and increasing insulin sensitivity in peripheral tissues as incretin hormone $[29,30]$. While effective, exendin-4 has some impediments such as still short-half life, low efficacy, and frequent administration for widespread clinical application.

Current trends in diabetes treatment using incretin hormones field include strategies to induce long-term therapeutic period and to increase efficacy. Several groups have explored alternative strategies to bypass the limitations of incretin hormones in vivo [16,31-33]. Among these methods, gene therapy provides a promising approach to regulate stability and efficacy of exendin- 4 .

In this study, we newly constructed chimeric plasmid expression vector encoding secretable exendin- 4 into one plasmid to improve efficiency and efficacy of TSTA system using two plasmids as polymeric-plasmid based gene therapy. Dendritic ABP-conjugated PAMAM (PAM-ABP) bioreducible polymer was designed as efficient gene delivery carrier. Of course, there was no doubt that $\mathrm{ABP}$ cationic polymer has high transfection efficiency, good biocompatibility and no toxicity as intracellular gene delivery carrier. However, to improve the use of ABP in vivo, ABP polymer was developed as PAM-ABP. PAM-ABP polymer can form compact polyplex to prevent decreased transfection efficiency by premature cleavage and gene release at early time [22], leading to much higher transfection efficiency to target site (Supplementary Figure 1).These systems with chimeric DNA and PAM-ABP polymer at 1:10 $w / w$ ratio made exendin- 4 to render the induction of high expression compared with existing TSTA system and $\mathrm{ABP}$ polymer without sever cytotoxicity, leading to the induction of increased insulin secretion in pancreatic $\beta$-cells (Figure 2). These results reaffirmed superior gene transfer ability of dendritic PAMABP bioreducible polymer and efficient exendin- 4 expression of chimeric TSTA DNA system.

The low cytotoxicity of bioreducible polymer including $\mathrm{ABP}$ and PAM-ABP compared with $25 \mathrm{~K}$ PEI causes that the disulfide bonds in their structure is degraded into non-toxic small molecules in reductive environment such as cytoplasm in cells (Supplementary Figure 2) $[22,24,28]$. It may be a possibility that PAM-ABP which has higher molecular weight than $\mathrm{ABP}$ polymer can induce a little cytotoxicity than $\mathrm{ABP}$ polymer by degraded single block. On the other hand, this may suggest another possibility that PAM-ABP polyplex will provide more long-term stability of polyplex in bloodstream due to the concentration difference of reducing molecular between in extracellular environment and in cytosol $[34,35]$, leading to increase in vivo efficacy due to the stability improvement of polyplex.

Next, we studied the possible mechanism for the effects of exendin-4 on insulin secretion in $\beta$-cells (Figure 3 ). Exendin- 4 stimulates insulin secretion form $\beta$-cells. Activation of GLP-1 receptor by exendin- 4 induces the impulsion of intracellular signaling pathway. cAMP is well-known as the main mediator of exendin-4 action on molecular functions for insulin secretion in $\beta$-cells [36]. PKA is also the primary mediator and a key component in the regulation of insulin secretion by cAMP activation. After all, cAMPPKA signaling in $\beta$-cells activated via exendin- 4 is one of the major pathway to induce the insulin secretion $[6,26]$. In NIT-1 insulinoma cells treated with $\mathrm{PAM}-\mathrm{ABP} /$ chimeric DNA system, exendin-4 expressed by PAM-ABP/chimeric DNA polyplex induced increased activation of PKA downstream signal response to up-regulation on upstream exendin-4-induced cAMP signaling pathway of $\beta$-cells. Taken together, our results indicate that exendin- 4 activates $\beta$-cell function via the stimulation of signaling pathway in cells to produce insulin. Importantly, these results provide an insight into therapeutic 
Citation: Kim PH, Lee M, Nam K, Kim SW. Enhanced Incretin Effects of Exendin-4 Expressing Chimeric Plasmid Based On Two-Step Transcription Amplification System with Dendritic Bioreducible Polymer for the Treatment of Type 2 Diabetes. J Gene Ther 2013;1(1): 9.

mechanisms of exendin-4 expressed by our newly improved exendin-4 expression system with PAM-ABP polymer.

Type 2 diabetes is a metabolic disorder that is characterized by high blood glucose. Also, insulin resistance is a representative trademark of type 2 diabetes and it often precedes the onset of hyperglycemia and indicates the elevations of lipid level in body.

First, we examined whether or not exendin-4 produced by various compounding with exendin-4 expression systems and several polymers actually has improved anti-diabetic effects. Figure $4 \mathrm{~A}$ showed that exendin-4 delivered by chimeric DNA system and dendritic $\mathrm{PAM}-\mathrm{ABP}$ cationic polymer induces greater glucoregulatory effects in diabetic mice. In current clinical field, two kinds of exendin4 -associated therapeutic agents including recently FDA approval agent have used [3]. However, they need twice a daily or once a week administration to maintain the normal glucose levels or need more high doses, although they show good effects. More long-acting system is required. In that point of view, our systems based on polymeric gene therapy showed long-lasting effects to lower high blood glucose via once intravenous injection. PAM-ABP/chimeric DNA system reached to normoglycemia within 3 days after injection and then maintained low glucose levels to total experiment time without hypoglycemia. More high improvements of anti-diabetic effects may be induced via the control of therapeutic DNA or polymer dose or the number of injection time.

The anti-hyperglycemic effects of incretin hormones are mediated by the key actions of enhancement of glucose-dependent insulin secretion $[7,37]$. Our results demonstrate that exendin- 4 expressed by chimeric DNA system with PAM-ABP can induce enhancement of insulin secretion and has insulin secretion-inducing ability in diabetic mice (Figure 5B). Taken together, these results in Figure 4A and $4 \mathrm{~B}$ showed an interactive function by glucose-dependent insulin secretion and insulin-mediated glucose clearance by exendin- 4 .

Furthermore, incretin effects by exendin- 4 were dependent on exendin- 4 expression. The reduction of exendin- 4 expression leaded to the decrease of glucoregulatory and insulinotropic effects, correlating with returns to high glucose values and low insulin levels in blood (Figure 4). After all, long-lasting expression of endogenous exendin- 4 by chimeric DNA system and PAM-ABP dendritic polymer leads to improved and extended anti-diabetic effects of exendin- 4 as incretin hormone against diabetes.

In obese patient with type 2 diabetes, lipolysis is increased because adipose tissue is resistance to insulin action [38]. Consequently, elevations of FFA levels by lipolysis bring about insulin resistance, leading to a decrease insulin-stimulated glucose uptake. Finally, elevated levels of FFA in blood are suggested one of the reasons inducing low incretin effects. Our study have shown that elevated basal FFA levels were reduced by the administration of PAM-ABP/ chimeric DNA polyplex (Figure 5A). In addition, glucose levels and insulin production by $\mathrm{PAM}-\mathrm{ABP} /$ chimeric DNA polyplex were observed the best results among mice treated with various compounding when decreased FFA levels. Some reports are consistent with our results that reducing FFA levels in blood improves glucose uptake and insulin sensitivity $[27,39,40]$.

Moreover, elevations of FFA level also cause increased lipolysis of adipocyte by obese [39]. Consequently, elevated FFA may produce insulin resistance as well as FFA-induced $\beta$-cells apoptosis. However,
Minhyung Lee, Liu L or JH Jeong et al. demonstrated the ability of exendin- 4 to protect from FFA-induced or hypoxia-induced $\beta$-cell apoptosis $[15,23,41]$. Thereby, exendin- 4 expressed by chimeric DNA and PAM-ABP dendritic polymer may protect $\beta$-cells from FFAinduced cytotoxicity, resulting in improvement of insulinotropic effects and enhanced glucose disposal in diabetic mice. In addition, our system will be able to help avoid lipotoxicity by high FFA elevations.

Also, the systemic administration of PAM-ABP/chimeric DNA polyplex can lead to any toxicity by excessive liver uptake of polyplex. It is well-known that the size of particles below $100 \mathrm{~nm}$ can pass from blood to liver sinusoids [42]. Our polyplex has the size below $80 \mathrm{~nm}$. This is enough size capable of passing into liver. However, the results in Figure 5B showed the reduced liver injury of PAM-ABP/chimeric DNA polyplex via ALT and AST levels which are a representative enzyme indicating liver toxicity. This means that PAM-ABP and chimeric DNA used did not induce sever hepatic toxicity. All taken together, these data demonstrate that exendin- 4 expressed by PAM$\mathrm{ABP} /$ chimeric DNA polyplex induced the prolonged and potentincretin effects than those of TSTAT system.

Here we showed the ability of exendin- 4 by chimeric TSTA pDNA system and PAM-ABP dendritic polymer for the treatment of type 2 diabetes. PAM-ABP/Chimeric DNA system showed an increased exendin- 4 expression and insulin induction. Moreover, treatment of PAM-ABP/chimeric DNA polyplex to pancreatic $\beta$-cells increased cAMP levels for insulin secretion. Consequently, exendin4-stimulated cAMP up-regulation induced the activation of increased PKA on downstream signaling pathway, leading to an increased insulin production. Based on these in vitro results, diabetic mice treated with $\mathrm{PAM}-\mathrm{ABP} /$ chimeric DNA polyplex also showed greater normalization of hyperglycemia and improved insulinotropic effects by increased exendin- 4 levels in serum. Furthermore, diabetic mice treated with $\mathrm{PAM}-\mathrm{ABP} /$ chimeric DNA polyplex showed the reduced FFA level in blood as well as no serious liver toxicity associated with polyplex infusion compared with $25 \mathrm{~K} \mathrm{PEI/chimeric} \mathrm{DNA} \mathrm{polyplex-}$ treated mice. Chimeric TSTA plasmid condensation via PAMABP dendritic cationic polymer delineate therapeutic potential of polymeric based gene therapy using efficient gene expression system and superior gene delivery carrier, enabling improve anti-diabetic effects.

\section{References}

1. Kim SW (2011) Polymeric gene delivery for diabetic treatment. Diabetes Metab J 35: 317-326.

2. Kim PH, Kim SW (2012) Polymer-based delivery of glucagon-like Peptide-1 for the treatment of diabetes. ISRN Endocrinol 2012: 340632

3. Scott LJ (2012) Exenatide extended-release: a review of its use in type 2 diabetes mellitus. Drugs 72: 1679-1707.

4. Association AD (2012) Diagnosis and classification of diabetes mellitus Diabetes Care 1: S64-S71.

5. Krol S, Ellis-Behnke R, Marchetti P (2012) Nanomedicine for treatment of diabetes in an aging population: state-of-the-art and future developments. Nanomedicine 1: S69-S76.

6. Verspohl EJ (2009) Novel therapeutics for type 2 diabetes: incretin hormone mimetics (glucagon-like peptide-1 receptor agonists) and dipeptidyl peptidase-4 inhibitors. Pharmacol Ther 124:113-138.

7. Drucker DJ (2003) Enhancing incretin action for the treatment of type 2 diabetes. Diabetes Care 26: 2929-2940. 
Citation: Kim PH, Lee M, Nam K, Kim SW. Enhanced Incretin Effects of Exendin-4 Expressing Chimeric Plasmid Based On Two-Step Transcription Amplification System with Dendritic Bioreducible Polymer for the Treatment of Type 2 Diabetes. J Gene Ther 2013;1(1): 9.

8. Arnes L, Moreno P, Nuche-Berenguer B, Valverde I, Villanueva-Penacarrillo ML (2009) Effect of exendin-4 treatment upon glucose uptake parameters in rat liver and muscle, in normal and type 2 diabetic state. Regul Pept 153: 8892.

9. Ahren B (2011) The future of incretin-based therapy: novel avenues--nove targets. Diabetes ObesMetab 1:158-166.

10. Bose AK, Mocanu MM, Carr RD, Brand CL, Yellon DM (2005) Glucagon-like peptide 1 can directly protect the heart against ischemia/reperfusion injury. Diabetes 54: 146-151.

11. Martin B, Golden E, Carlson OD, Pistell P, Zhou J, et al. (2009) Exendin-4 improves glycemic control, ameliorates brain and pancreatic pathologies, and extends survival in a mouse model of Huntington's disease. Diabetes 58: 318-328.

12. Mikhail N (2008) Incretin mimetics and dipeptidyl peptidase 4 inhibitors in clinical trials for the treatment of type 2 diabetes. Expert Opin Investig Drugs 17: 845-853

13. Voutetakis A, Cotrim AP, Rowzee A, Zheng C, Rathod T, et al. (2010) Systemic delivery of bioactive glucagon-like peptide 1 after adenoviralmediated gene transfer in the murine salivary gland. Endocrinology 151: 4566-4572.

14. Choi SH, Lee HC (2011) Long-term, antidiabetogenic effects of GLP-1 gene therapy using a double-stranded, adeno-associated viral vector. Gene Ther 18: $155-163$

15. Jeong JH, Yook S, Jung $Y$, Im BH, Lee M, et al. (2012) Functional enhancement of beta cells in transplanted pancreatic islets by secretion signal peptide-linked exendin-4 gene transduction. J Control Release 159 368-375.

16. Pan CQ, Buxton JM, Yung SL, Tom I, Yang L, et al. (2006) Design of a long acting peptide functioning as both a glucagon-like peptide-1 receptor agonis and a glucagon receptor antagonist. J Biol Chem 281: 12506-12515.

17. Li Y, Zheng X, Tang L, Xu W, Gong M (2011) GLP-1 analogs containing disulfide bond exhibited prolonged half-life in vivo than GLP-1. Peptides 32 1303-1312.

18. Lee J, Lee C, Kim TH, Lee ES, Shin BS, et al. (2012) Self-assembled glycol chitosan nanogels containing palmityl-acylated exendin-4 peptide as a longacting anti-diabetic inhalation system. J Control Release 161:728-734

19. Choi S, Oh S, Lee M, Kim SW (2005) Glucagon-like peptide-1 plasmid construction and delivery for the treatment of type 2 diabetes. Mol Ther 12 885-891.

20. Kim TI, Lee M, Kim SW (2012) Efficient GLP-1 gene delivery using two-step transcription amplification plasmid system with a secretion signal peptide and arginine-grafted bioreducible polymer. J Control Release 157: 243-248.

21. Kim PH, Lee M, Kim SW (2012) Delivery of two-step transcription amplification exendin-4 plasmid system with arginine-grafted bioreducible polymer in type 2 diabetes animal model. J Control Release162: 9-18.

22. Nam HY, Nam K, Lee M, Kim SW, Bull DA (2012) Dendrimer type bioreducible polymer for efficient gene delivery. J Control Release160: 592-600.

23. Ah Kim H, Lee S, Park JH, Lee S, Lee BW, et al. (2009) Enhanced protection of Ins-1 beta cells from apoptosis under hypoxia by delivery of DNA encoding secretion signal peptide-linked exendin-4. J Drug Target 17: 242-248.

24. Kim TI, Ou M, Lee M, Kim SW (2009) Arginine-grafted bioreducible poly (disulfide amine) for gene delivery systems. Biomaterials 30: 658-664.

25. Yan Y, Such GK, Johnston AP, Best JP, Caruso F (2012) Engineering particles for therapeutic delivery: prospects and challenges. ACS Nano 6 3663-3669.

26. Yu Z, Jin T (2010) New insights into the role of cAMP in the production and function of the incretin hormone glucagon-like peptide-1 (GLP-1). Cell Signal 22: 1-8.

27. Boden $\mathrm{G}$ (2002) Interaction between free fatty acids and glucose metabolism Curr Opin Clin Nutr Metab Care 5: 545-549.
28. Kim PH, Kim J, Kim TI, Nam HY, Yockman JW, et al. (2011) Bioreducible polymer-conjugated oncolytic adenovirus for hepatoma-specific therapy via systemic administration. Biomaterials 32: 9328-9342.

29. Gavin JR $3^{\text {rd }}$ (2012) Initiating a glucagon-like peptide-1 receptor agonist in the management of type 2 diabetes mellitus. J Am Osteopath Assoc 112: S16-21.

30. Meier JJ (2012) GLP-1 receptor agonists for individualized treatment of type 2 diabetes mellitus. Nat Rev Endocrinol 8: 728-742.

31. Kim JG, Baggio LL, Bridon DP, Castaigne JP, Robitaille MF, et al. (2003) Development and characterization of a glucagon-like peptide 1-albumin conjugate: the ability to activate the glucagon-like peptide 1 receptor in vivo. Diabetes 52: 751-759.

32. Gaddy DF, Riedel MJ, Bertera S, Kieffer TJ, Robbins PD (2012) dsAAV8mediated gene transfer and beta-cell expression of IL-4 and beta-cell growth factors are capable of reversing early-onset diabetes in NOD mice. Gene Ther 19: 791-799.

33. Jean M, Alameh M, De Jesus D, Thibault M, Lavertu M, et al. (2012) Chitosan-based therapeutic nanoparticles for combination gene therapy and gene silencing of in vitro cell lines relevant to type 2 diabetes. Eur J Pharm Sci 45:138-149.

34. Son S, Namgung R, Kim J, Singha K, Kim WJ (2012) Bioreducible polymers for gene silencing and delivery. Acc Chem Res 45:1100-1112.

35. Cheng R, Feng F, Meng F, Deng C, Feijen J, et al. (2011) Glutathioneresponsive nano-vehicles as a promising platform for targeted intracellular drug and gene delivery. J Control Release 152: 2-12.

36. Doyle ME, Egan JM (2007) Mechanisms of action of glucagon-like peptide 1 in the pancreas. Pharmacol Ther 113: 546-593.

37. Davidson JA, Parente EB, Gross JL (2008) Incretin mimetics and dipeptidy peptidase-4 inhibitors: innovative treatment therapies for type 2 diabetes. Arq Bras Endocrinol Metabol 52: 1039-1049.

38. Delarue J, Magnan C (2007) Free fatty acids and insulin resistance. Curr Opin Clin Nutr Metab Care 10: 142-148.

39. Schenk S, Saberi M, Olefsky JM (2008) Insulin sensitivity: modulation by nutrients and inflammation. J Clin Invest 118: 2992-3002

40. Salgin B, Ong KK, Thankamony A, Emmett P, Wareham NJ, et al. (2012) Higher fasting plasma free Fatty Acid levels are associated with lower insulin secretion in children and adults and a higher incidence of type 2 diabetes. $J$ Clin Endocrinol Metab 97: 3302-3309.

41. Liu L, Wang Y, Wang L, Lin Y, Liu X, et al. (2012) Exendin-4 Protects Murine Pancreatic beta-Cells from Free Fatty Acid-Induced Apoptosis through PI-3K Signaling. Endocr Res 38: 40-47.

42. Wisse E, Jacobs F, Topal B, Frederik P, De Geest B (2008) The size of endothelial fenestrae in human liver sinusoids: implications for hepatocytedirected gene transfer. Gene Ther 15: 1193-1199.

\section{Acknowledgements}

This work was supported by NIH DK077703, USA (SWK) and WCU 200900000000024, the Ministry of Education, Science and Technology, Korea, and the grant (2012K001394) (MHL) from Ministry of Education, Science and Technology in Korea. 